\title{
Lenguaje, aprendizaje de lenguas extranjeras, inglés e interculturalidad: una perspectiva ecuatoriana
}

\section{Language, foreign language learning, English, and interculturality: An Ecuadorian perspective}

Fabián Darío Rodas Pacheco

Universidad de Cuenca, Cuenca, Ecuador

fabian.rodas@ucuenca.edu.ec

https://orcid.org/0000-0001-8263-5015

Elisabeth Luisa Rodas Brosam

Universidad de Cuenca, Cuenca, Ecuador

elisabeth.rodas@ucuenca.edu.ec

https://orcid.org/0000-0002-6518-6243

Recepción: 19/03/2021 | Aceptación: 18/05/2021 | Publicación: 10/09/2021

Cómo citar (APA, séptima edición):

Rodas-Pacheco, F. D., Rodas-Brosam, E. L. (2021). Lenguaje, aprendizaje de lenguas extranjeras, inglés e interculturalidad: una perspectiva ecuatoriana. INNOVA Research Journal, 6(3), 1-16. https://doi.org/10.33890/innova.v6.n3.2021.1726

\section{Resumen}

El lenguaje, como parte de la vida cotidiana de los individuos, ha sido definido y redefinido constantemente de varias maneras, permitiendo conocer el contexto local que les rodea al igual que el mundo exterior. Por otro lado, con el pasar de los tiempos, el lenguaje, a nivel global, se ha constituido en parte esencial del currículo oficial educativo y, en las últimas décadas, parte de la enseñanza y aprendizaje de lenguas extranjeras. El objetivo de esta revisión bibliográfica descriptiva es compartir una serie de elementos y conceptos para complementar o reforzar el conocimiento actual de los lectores sobre el lenguaje y su rol en la enseñanza y aprendizaje de idiomas extranjeros, así como también la importancia del inglés y su nexo con la interculturalidad hoy en día. Se consideró principalmente las fuentes primarias y secundarias directamente relacionadas con los temas tratados (periodo 2000-2020) y aquellas obras de autores cuyo trabajo seminal ha aportado al desarrollo del área disciplinar. El lenguaje, como medio de comunicación humana, permite desarrollar la competencia comunicativa, pero lo más importante, da paso a que 
los individuos se conozcan y se relacionen de una mejor manera con las personas que habitan a su alrededor.

Palabras claves: lenguaje; idiomas extranjeros; inglés; interculturalidad.

\begin{abstract}
Language, as part of individuals' everyday lives, has been defined and redefined constantly in different ways, making it possible to know the local context that surrounds them as well as the outside world. On the other hand, with the passing of time, language, at a global level, has become an essential part of the official educational curriculum and, in recent decades, part of the teaching and learning of foreign languages. The purpose of this descriptive literature review is to share a series of elements and concepts to complement or reinforce readers' current knowledge about language and its role in the teaching and learning of foreign languages, as well as the importance of English and its link with interculturality today. Primary and secondary sources were considered if directly connected to the topic under review (between 2000-2020) and authors whose seminal work has contributed to the disciplinary field. Language, as a means of human communication, allows the development of communicative competence, but more importantly, it gives way for individuals to know and interact in a better way with the people who live around them.
\end{abstract}

Keywords: language; foreign languages; English; interculturality.

\title{
Introducción
}

El lenguaje como tema de estudio, posiblemente más que cualquier otra área de contenido, se redefine constantemente. Es la herramienta que todo el mundo utiliza en el aprendizaje y la enseñanza, pero que resulta difícil definirla por sí misma. En cuanto a la enseñanza de idiomas extranjeros, es importante mencionar que, a diferencia de otras materias del plan de estudios, no ha tenido un hogar disciplinario consistente en el cual anclar sus contenidos o teorías de aprendizaje, enseñanza y conocimiento o dominio. Por otro lado, los lingüistas ofrecen una importante base disciplinaria, pero las descripciones lingüísticas de los idiomas como asignaturas, por muy útiles que sean, no definen lo que se debería enseñar en el aula de idiomas extranjeros (Larsen-Freeman y Freeman, 2008).

Por lo tanto, para que un docente de lengua extranjera pueda tomar decisiones adecuadas con respecto a su práctica docente diaria necesita tener una perspectiva amplia del lenguaje en general, el inglés como lengua extranjera en particular y la interculturalidad como herramienta de conexión no solo con miembros de la cultura local sino también con individuos alrededor del mundo. En este contexto, el objetivo de esta revisión bibliográfica descriptiva es compartir una serie de elementos y conceptos para complementar o reforzar el conocimiento actual de los lectores sobre el lenguaje y su rol en la enseñanza y aprendizaje de idiomas extranjeros, así como también la importancia del inglés y su nexo con la interculturalidad hoy en día. Este conocimiento busca que se establezcan interacciones interculturales en este mundo globalizado, dando valor a la cultura propia y la foránea, propiciando intercambios más equitativos (Byram, 1977).

En este contexto, esta revisión de la literatura se divide en cinco secciones: La primera parte pone en consideración varios aspectos y conceptos sobre el lenguaje en general y la segunda se enfoca en la importancia del lenguaje en la enseñanza y aprendizaje de idiomas extranjeros. En 
la tercera sección se habla sobre el inglés en la actualidad y cuál es su importancia en el aula de clase. Como cuarto punto se cubre el tema del inglés y la interculturalidad y se culmina con el proceso de enseñanza y aprendizaje del inglés en el contexto ecuatoriano. Finalmente, esperamos que los resultados de este trabajo contribuyan con los actuales debates del campo de la enseñanza de lenguas extranjeras.

\section{Métodos}

Una revisión de la literatura es pertinente cuando se requiere recolectar información de varias fuentes, lo que permitirá, de una manera objetiva, proveer al lector una serie de cosmovisiones que guíen su entendimiento sobre ciertos temas a tratar. En este contexto, este estudio se enmarca dentro de la metodología de revisión bibliográfica descriptiva. Day (2005) recuerda que un artículo de revisión bibliográfica puede hacer referencia a cualquier tema, pues revisa los trabajos recientes de un área determinada, o los trabajos de uno o varios autores. Así mismo, un artículo de este tipo "tiene por objetivo resumir, analizar, evaluar o sintetizar información ya publicada” (Day, 2005, p. 13). Para realizar esta revisión bibliográfica descriptiva se consideró principalmente las fuentes primarias directamente relacionadas con los temas de lenguaje, interculturalidad e inglés como lengua extranjera y aquellas obras de autores cuyo trabajo seminal ha aportado al desarrollo del área disciplinar. Se delimitó la revisión sistemática de las fuentes primarias y secundarias entre los años 2000-2020, a excepción de las obras seminales.

\section{Resultados}

\section{El lenguaje}

La interacción del lenguaje con la vida social es vista como un asunto de acción humana, basada en conocimiento, algunas veces consciente, a menudo inconsciente, lo que les permite a las personas utilizar el idioma (Hymes, 1972). Este autor también manifiesta que los eventos del habla y otros sistemas más complejos se caracterizan por tener propiedades que no se reducen necesariamente a aquellas que tienen que ver con la competencia del habla de las personas. Dicha competencia, sin embargo, subyace en la conducta comunicativa, no solamente dentro de las comunidades sino en encuentros entre ellas. La competencia del habla puede ser vista como parte de una serie de sistemas de encuentro en niveles de diferente alcance (Hymes, 1972).

Por su parte, Duranti (1989) se refiere a la etnografía del habla y menciona que ésta estudia el uso del lenguaje como se presenta en la vida cotidiana de comunidades particulares. Según el autor, el método etnográfico está apoyado por técnicas desarrolladas en otras áreas de estudio, tales como la pragmática, el análisis conversacional, la poética y la historia. En este escenario, desde el punto de vista del contenido de la interacción verbal diaria, la etnografía se interesa en la relación entre el uso del lenguaje y los sistemas locales de conocimiento y conducta social; por lo tanto, analiza al habla como uno de los puntos más importantes para la creación, recreación y transmisión de patrones culturales de conocimiento y acción social. Asimismo, para la etnografía es relevante lo que se logra a través del habla y cómo el discurso está relacionado y construido por medio de aspectos de la organización social y de las presunciones, valores y creencias alrededor del mundo. 
Con respecto a lenguaje, el Merriam-Webster's Collegiate Dictionary (2003) lo define como "un medio sistemático de comunicar ideas o sentimientos a través del uso convencional de signos, sonidos, gestos, señas que tienen un significado sobreentendido" (p. 699). Pinker (1994) va más allá de la definición anterior al considerar que, aunque el lenguaje es una destreza compleja y especializada, "se desarrolla espontáneamente en el niño, sin esfuerzo consciente o instrucción formal" y además "se desarrolla inconscientemente de su lógica subyacente, es cualitativamente similar en todos los individuos, y es diferente de las habilidades más generales para procesar información o comportarse inteligentemente" (p. 18). Por otro lado, Scollon (2004) sostiene que el lenguaje es un fenómeno variado y complejo que no se presenta en unidades claramente definidas.

Finalmente, para Brown (2007) el concepto de lenguaje incluye los siguientes aspectos: 1) es sistemático; 2) incluye un grupo de símbolos arbitrarios que 3) son principalmente vocales pero también pueden ser visibles; 4) los símbolos generalmente tienen significados convencionales a los que hacen referencia; 5) el lenguaje es usado para la comunicación; 6) opera en una comunidad lingüística o cultura; 7) es esencialmente humano pero posiblemente no limitado a él; y 8) todas las personas lo adquieren de la misma manera ya que tanto el lenguaje como su aprendizaje es universal.

Una vez que se han considerado algunas características generales del lenguaje, a continuación, se abordará el aprendizaje de idiomas extranjeros.

\section{El proceso de enseñanza y aprendizaje de idiomas extranjeros}

Para aprender una lengua extranjera es necesario utilizar el idioma meta en forma significativa, como un vehículo que conduzca a alcanzar una mejor comprensión tanto de la propia cultura como de la ajena (Ballestero y Batista, 2007). Por su parte, Brown (2007) manifiesta que el aprendizaje de un idioma extranjero no es tarea sencilla, pues la persona se ve afectada a medida que lucha por ir más allá de los confines de su propia lengua y busca adentrarse en la nueva, en una nueva cultura, en una nueva forma de pensar, de sentir y de actuar. En este contexto, el autor manifiesta que, a más de existir muchas variables en el proceso de aprendizaje, se requiere de un compromiso grande, una participación completa, una respuesta física, intelectual y emocional total para poder enviar y recibir mensajes en otro idioma exitosamente. Así, el aprendizaje de otro idioma no se da de forma lineal por medio de un grupo de pasos sencillos, sino que involucra diferentes factores. Por lo que ciertos cursos para el aprendizaje de idiomas extranjeros son a menudo inadecuados campos de entrenamiento en sí mismos que impiden un aprendizaje exitoso. En este sentido, son muy pocas las personas que, tan sólo en el aula de clases, logran fluidez en otro idioma (Brown, 2007).

Por otro lado, no todos los lingüistas e investigadores que se dedican al aprendizaje de otro idioma están de acuerdo sobre cómo se adquiere otra lengua. No obstante, hay ciertos patrones históricos que han surgido, resaltando tendencias y modas en su adquisición, que podrían ser brevemente resumidos en las siguientes escuelas de pensamiento. Brown (2007) manifiesta que en los años cuarenta y cincuenta, la escuela de la lingüística estructural / descriptiva con sus defensores Leonard Bloomfield, Edward Sapir, Charles Hockett, Charles Fries y otros, se 
enorgullecían en una rigurosa aplicación de observaciones científicas de los idiomas humanos. En este escenario, Brown sostiene que tan solo respuestas públicamente observables podían estar sujetas a investigación. Por lo tanto, la tarea del lingüista, de acuerdo con los estructuralistas, consistía en describir los idiomas humanos e identificar las características estructurales de éstos.

Adicionalmente, en la década de los sesenta, los lingüistas generativo- transformacionales aparecieron a través de la influencia de Noam Chomsky y de sus seguidores (Brown, 2007). Este autor indica que Chomsky trataba de demostrar que el lenguaje humano no podía ser examinado tan solo en términos de estímulo y respuesta observables. Por el contrario, el lingüista generativo no solo estaba interesado en la descripción del lenguaje, sino en llegar a un nivel de explicación adecuado de este. En esta misma línea de pensamiento, los psicólogos cognitivos afirmaban que, significado, comprensión y conocimiento constituían datos significativos para estudios de su área; por lo tanto, en lugar de concentrarse mecánicamente en las conexiones estímulo-respuesta, los cognitivistas trataban de descubrir principios psicológicos de organización y funcionamiento.

Finalmente, Brown (2007), con respecto al constructivismo, afirma que difícilmente es una nueva escuela de pensamiento y que nombres como los de Jean Piaget y Lev Vigotsky a menudo están asociados con esta escuela y con el estudio de idiomas. El constructivismo apareció como un paradigma prevaleciente a finales del siglo XX y, hoy por hoy, es una ortodoxia. Una característica importante del constructivismo es que integra los paradigmas linguí́stico, psicológico y sociológico en uno solo, diferente a lo que sucedía en el siglo XIX. El constructivismo pone énfasis en el aspecto cognitivo y social. Para el primero, el énfasis subyace en el hecho de que el estudiante construye su propia representación de la realidad. A su vez, enfatiza la importancia de la interacción y del aprendizaje cooperativo en la construcción de imágenes de la realidad tanto cognitivas como emocionales (Brown, 2007). Adicionalmente, investigadores dedicados al estudio del aprendizaje de la lengua materna y de un segundo idioma han adelantado esta perspectiva constructivista llevando a cabo estudios del discurso en el aula, de los factores socioculturales en el aprendizaje y sosteniendo teorías interaccionistas.

Brown (2007) resume las tres escuelas de pensamiento sobre la adquisición de otro idioma en el siguiente cuadro:

\section{Tabla 1}

Escuelas de pensamiento en la adquisición de otro idioma

\begin{tabular}{lll}
\hline Período de Tiempo & Escuela de Pensamiento & Temas Característicos \\
\hline Inicios de 1900, 1940 y & Lingüística Estructurada y & Descripción \\
1950 & Psicología Conductual & Comportamiento observable \\
& & Método Científico \\
& & Empirismo \\
& & Estructura Superficial \\
& Condicionamiento \\
& & Refuerzo \\
\hline 1960,1970 y 1980 & Gramática Generativa y Psicología & Gramática Generativa \\
& Cognitiva & Adquisición, Innato \\
\hline
\end{tabular}




\begin{tabular}{|c|c|c|}
\hline \multirow[t]{6}{*}{ Período de Tiempo } & Escuela de Pensamiento & Temas Característicos \\
\hline & & Interlengua \\
\hline & & Sistematicidad \\
\hline & & Gramática Universal \\
\hline & & Competencia \\
\hline & & Estructura Profunda \\
\hline \multirow[t]{7}{*}{1980,1990 y 2000} & Constructivismo & Discurso Interactivo \\
\hline & & Variables Socioculturales \\
\hline & & Aprendizaje Cooperativo \\
\hline & & Aprendizaje basado en el \\
\hline & & Descubrimiento \\
\hline & & Construcción de Significado \\
\hline & & Variabilidad en la interlengua \\
\hline
\end{tabular}

Fuente: Brown (2007)

Como se puede observar, a través de los años, el aprendizaje de una lengua extranjera ha atravesado por diferentes tendencias y modas en su adquisición que han puesto de relieve una variedad de enfoques y formas de aprender otro idioma. Sin duda, de acuerdo a las nuevas necesidades y desafíos que se presenten a futuro, se generarán aún nuevas formas de aprendizaje.

\section{La internacionalización del inglés}

McKay (2002) sostiene que, contrariamente a lo que la mayoría de personas piensan, un idioma internacional no es solamente aquel que posee una gran cantidad de hablantes nativos. En realidad, para que un idioma sea internacional se requiere que no esté ligado tan solo a una cultura o nación, sino a lugares en donde sirva tanto para necesidades globales como locales, constituyéndose de esta manera en un idioma de amplio uso.

En este contexto, Seidlhofer (2004) indica que, en la actualidad, el inglés en el mundo se encuentra en un estado de balance delicado, o según los físicos, en un equilibrio inestable. Esto se debe a que mientras la mayoría de usuarios del inglés se encuentran localizados en países donde este constituye una lengua extranjera, el control sobre las normas del lenguaje todavía está en poder de aquellas personas que lo utilizan como lengua materna. En este contexto, Beneke (1991) estima que alrededor del $80 \%$ de los intercambios verbales en los cuales el inglés es usado como lengua extranjera o como segunda lengua no involucra a ningún nativo hablante. Por otro lado, Graddol (1997) comenta que si bien es cierto que los hablantes nativos del inglés pueden considerar que el idioma les pertenece, sin embargo, serán aquellas personas que lo utilizan como segunda lengua o como lengua extranjera quienes determinarán su futuro mundial.

Aunque el inglés es la lengua materna o de uso oficial en por lo menos 60 países, Douglas (2014) recuerda que es una segunda lengua importante en muchos países más. Así mismo, menciona que hay más hablantes de inglés como segunda o lengua extranjera en todo el mundo que hablantes nativos del idioma. Es por ello que el inglés se ha convertido en lingua franca. Adicionalmente, de acuerdo a un reporte sobre el uso del inglés como medio de instrucción en países no nativo hablantes (Dearden, 2014), esta tendencia va en crecimiento. Así mismo, el idioma más comúnmente utilizado en las publicaciones científicas es el inglés, con la mayoría de las revistas científicas de prestigio publicadas en este idioma (van Weijen, 2012). 
Con el propósito de entender mejor el uso del inglés en los diferentes países, Kachru (1985) desarrolló el modelo de los tres círculos concéntricos de la lengua, que actualmente se mantiene como uno de los modelos más influyentes cuando se trata de agrupar las diferentes variedades de esta lengua en el mundo. Kachru (1985) describió “el tipo de esparcimiento, los patrones de adquisición y los dominios funcionales en los cuales el inglés es usado a lo largo y ancho de las culturas y los idiomas" (p. 2), mediante tres círculos concéntricos: el interno, el externo y el que está en expansión. El círculo interno representa los países de base inglesa tradicional, tales como el Reino Unido, Estados Unidos, Australia, Nueva Zelanda, Irlanda, la parte anglófona de Canadá y algunos territorios del Caribe. El número total de hablantes de inglés en el círculo interno es mayor de 380 millones. A su vez, el círculo externo incluye los países donde el inglés no es la lengua nativa, pero es importante por razones históricas y juega un papel relevante en las instituciones nacionales, tanto como lengua oficial como no oficial (e.g., India, Filipinas, Bangladesh, Pakistán, Malasia, Tanzania, Kenya, partes no anglófonas de Sudáfrica y Canadá). El número total de hablantes de inglés en el círculo externo está entre los 150 y los 300 millones. Finalmente, el círculo en expansión incluye a los países donde el inglés no representa un papel histórico ni gubernamental (e.g., China, Rusia, Japón, Corea, Egipto, Indonesia y la mayoría de los países europeos y latinoamericanos); no obstante, está muy extendido como lengua extranjera o lingua franca. El número total de hablantes en este círculo es difícil de establecer puesto que es empleado para fines específicos y limitados, normalmente Business English. Se estima un número de hablantes entre 100 millones y un billón (Kachru, 1985, 1992).

A continuación, se da a conocer el modelo de los círculos concéntricos de Kachru.

\section{Figura 1}

\section{Círculos concéntricos del inglés}

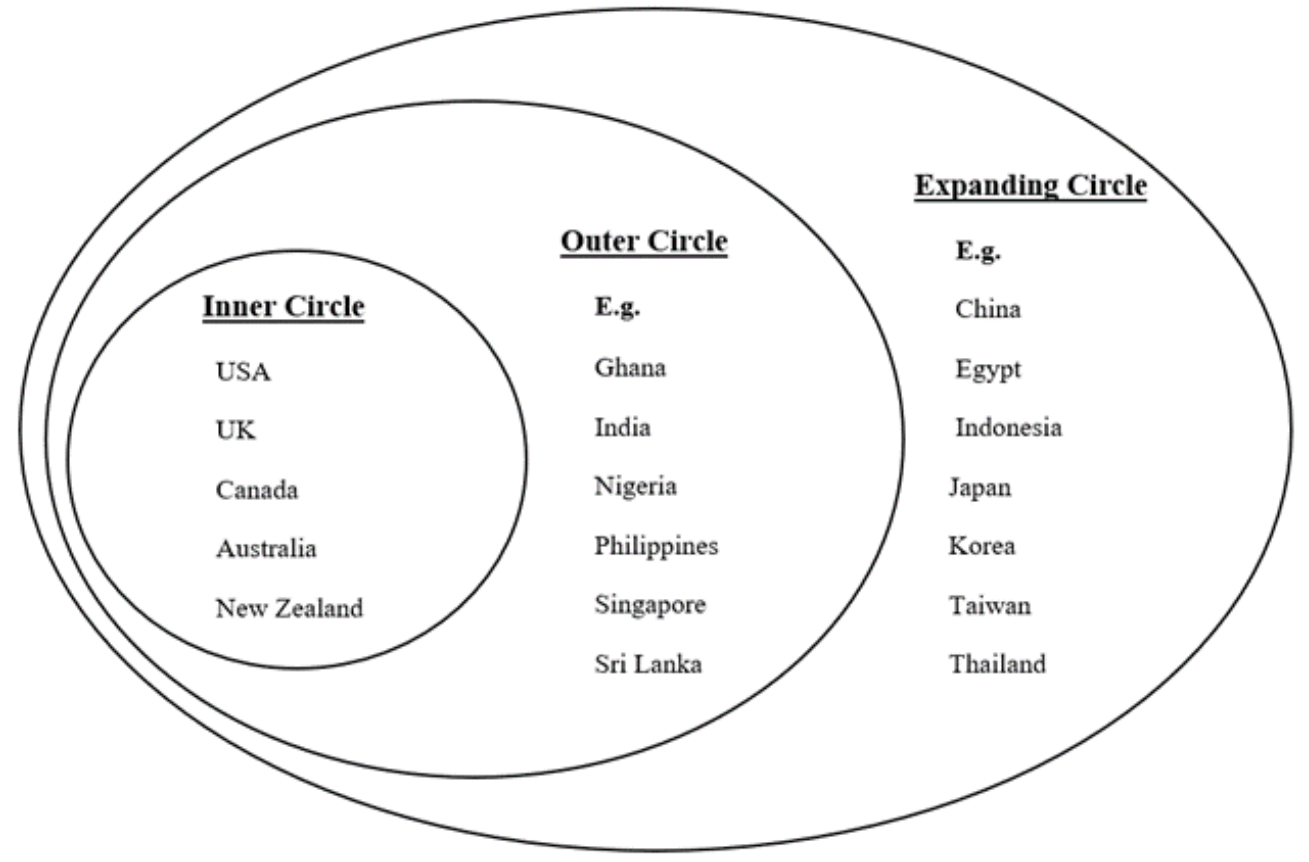

Nota: Kachru (1992) 
Sobre el modelo presentado por Kachru (1985), Jenkins (2008) indica que el círculo interior está representado por las personas que utilizan el inglés como lengua materna, el círculo del medio por aquellas personas que lo utilizan como segunda lengua o como lengua extranjera y el círculo exterior o de expansión por las personas que lo utilizan como lingua franca. Además, al ser más del $80 \%$ de personas que utilizan este idioma no nativo hablantes, ella manifiesta que serán ellos quienes ejercerán un gran impacto en el inglés en el futuro y que los países tales como los Estados Unidos y el Reino Unido no estarán en condiciones de establecer las pautas a seguir. Asimismo, de este $80 \%$ de hablantes no nativos, el grupo más grande está conformado por el círculo en expansión quienes utilizan el inglés como lingua franca.

Con respecto al término "lingua franca", tanto Samarin (1987) como House (1999) consideran que este hace referencia al uso de un idioma para la comunicación por personas con diferentes lenguas maternas y diferentes culturas, para quienes este lenguaje es considerado lengua extranjera o un segundo idioma. De acuerdo a esta definición, una lingua franca no tiene hablantes nativos. Otra definición sugiere que "el inglés como lingua franca puede incluir a los hablantes nativos; no obstante, en la mayoría de casos, es considerado como un idioma de contacto entre personas quienes no comparten ni un idioma común ni una cultura nacional común, y para quienes el inglés es un lenguaje adicional" (Firth,1996, p. 240).

Seidlhofer (2004) comenta que, por primera vez en la historia, un idioma ha alcanzado dimensiones globales reales; por lo tanto, se está configurando internacionalmente gracias no solamente a los hablantes nativos sino también a los hablantes no nativos. Este proceso se ha acelerado debido a la dramática expansión de la comunicación electrónica a través del internet. Adicionalmente, de acuerdo a Brumfit (2002), los miembros del círculo de expansión de Kachru (1985), quienes utilizan el inglés, constituyen un grupo cada vez más significativo que opera en una economía que va cobrando importancia y que ejerce un impacto en la economía de todas las naciones.

Sobre este mismo tema, Seidlhofer (2004) sostiene que otro factor que acelera el cambio en un idioma es el hecho de que los cambios generales en los entornos en los que se utiliza el inglés implican que el idioma es usado cada vez más por personas con variados niveles de dominio. Esto significa que los actos comunicativos ocurren entre personas que no tienen control de una gramática estandarizada y cuyo léxico y pronunciación no están de acuerdo con ninguna norma del idioma. Además, esta autora, en la misma línea que Samarin (1987) y House (1999), menciona que el inglés como lingua franca ha cobrado un estatus propio, independiente de cualquier norma establecida por los hablantes nativos, lo que garantiza su reconocimiento global.

Por otro lado, Seidlhofer (2004) argumenta que dondequiera que el inglés se utilice como la mejor opción para la comunicación entre personas que hablan diferentes lenguas, el inglés se convierte en un idioma internacional. En esta misma línea, ella comenta que el término "inglés internacional" es usado cuando hace referencia a dos situaciones que involucran la lengua y la cultura, mismas que son completamente diferentes. Por un lado, están los países del círculo externo de Kachru (1985), en donde el inglés es utilizado para cumplir con propósitos domésticos, intranacionales. Por otro lado, está el inglés como medio globalizador para la comunicación internacional, el cual va más allá de todas las fronteras nacionales. Finalmente, la autora indica 
que la diferencia entre lo local y lo global se pierde entre los círculos externo y de expansión, debido a que aquellas comunidades que utilizan el inglés internacionalmente en el círculo externo también participan en los usos globales de este idioma, tal como lo hacen los hablantes del círculo interno; por lo tanto, el inglés ha expandido su uso a través de todas las regiones que Kachru tan claramente ha definido.

Por su parte, Méndez García (2013) manifiesta que si bien es cierto que la globalización ha afectado negativamente a los países pobres en cuanto al aspecto económico se refiere, en el tema educativo, especialmente en lo que tiene que ver con la educación superior, ha dado lugar a que se generen un sinnúmero de programas que necesariamente vinculan a los estudiantes universitarios con contextos regionales y globales. De esta manera, se ha logrado mejorar el conocimiento en general y se han dado grandes pasos con respecto al conocimiento y comprensión del otro, es decir, en el campo de la interculturalidad. También la autora menciona que el contacto con contextos foráneos ha creado la necesidad de aprender nuevos idiomas, que permitan tanto a estudiantes como a docentes realizar estudios, intercambios y pasantías que sin duda alguna redundará en beneficio de sus respectivos perfiles profesionales.

Tener acceso a otros idiomas genera sensibilidad sobre las cosmovisiones de las personas y el relativismo de su forma de pensar: el estudio de idiomas puede revelar el relativismo de la forma en que las diferentes comunidades se conciben y se interpretan las unas a las otras. En esta línea de pensamiento, Domínguez Menéndez (2003) recuerda que la educación garantizará a los futuros profesionales la oportunidad de obtener empleos dignos que les permitan contribuir al desarrollo de sus pueblos; sin embargo, esto no se logrará si los gobiernos de turno no desarrollan políticas educativas tendientes a lograr este objetivo. Se requiere de políticas de Estado capaces de perdurar por sobre los cortos períodos de los gobiernos que tracen un proyecto por la excelencia, la equidad y la democracia en las aulas de clase.

\section{La enseñanza del inglés y la interculturalidad}

La educación intercultural, de acuerdo a lo que plantea Díaz-Aguado (2003), es una propuesta de acción educativa teórico-práctica; en ésta prevalece el reconocimiento de la existencia de los otros como sujetos poseedores de una cultura diferente y el conocimiento de lo que esto significa en términos de semejanzas y diferencias con la propia cultura escolar, que está caracterizada por múltiples influencias, lo que da lugar a un intercambio de valores para la emergencia de una situación más democrática y solidaria. En este escenario, López (2006) sugiere que, en el caso concreto de lo que ha venido a denominarse escuelas interculturales, abordar la diversidad cultural se plantea como una iniciativa o guía pedagógica ineludible, y en ningún caso como elemento perturbador de la vida escolar cotidiana.

Por su parte, Dimas (2016) manifiesta que un modelo de enseñanza intercultural debe tomar en cuenta el intercambio que se presenta entre estudiantes, el contexto de aprendizaje y las perspectivas del docente, especialmente hoy en día cuando los países están debatiendo el tema de la diversidad en este mundo globalizado. En este contexto, para que los estudiantes desarrollen la conciencia intercultural, los docentes de idiomas deberían utilizar el modelo de competencia comunicativa intercultural (CCI) propuesto por Byram (1997), que promulga la incorporación de la competencia intercultural a las competencias lingüística, sociolingüística y discursiva. 
Kumaravadivelu (2008) recuerda que "la cultura ha sido una parte integral de la enseñanza de idiomas desde tiempos inmemorables" (p. 23). También menciona que los profesores de idiomas extranjeros reconocieron la necesidad de enseñar la cultura como parte importante de la lengua después de la terminación de la Segunda Guerra Mundial, debido al comercio y a la comunicación internacional, cobrando mayor interés en los años 90 gracias a la migración masiva. Adicionalmente, Kumaravadivelu (2008) menciona que el Proyecto "Estándares Nacionales para la Educación en Lenguas Extranjeras" (NSFLE) de Estados Unidos, elaboró un documento que contemplaba los estándares para la enseñanza de lenguas extranjeras, que incluía cinco áreas que fueron conocidas como las cinco $\mathrm{C}$, pues se referían a: Comunicación, Culturas, Conexiones, Comparaciones y Comunidades. Este documento estaba compuesto por 11 estándares claramente definidos, de los cuales cinco pertenecían directamente al término cultura.

Por otro lado, Chahak y Basirizadeh (2012) sostienen que la importancia de enseñar un idioma extranjero radica en la creencia de que la cultura y el idioma están interconectados, pues definitivamente la premisa clave es que un idioma no puede enseñarse aisladamente de la cultura y viceversa. Un lenguaje meta se aprende tomando en cuenta las costumbres y los diferentes puntos de vista de los grupos sociales, mismos que cobran sentido a través de la lengua. Adicionalmente, Wei (2005) expresa que el lenguaje posee un doble sentido ya que es un medio de comunicación, pero también es portador de cultura. En este sentido, no es posible concebir un idioma sin cultura y cultura sin idioma.

Dentro del escenario global y de la relevancia que ha tomado el inglés para dar acceso a saberes formalizados, a varias nociones del mundo, de las culturas y de la sociedad (Ballestero y Batista, 2007), toma importancia la figura del nuevo docente de este idioma. Gao (2008) sostiene que un profesor de lenguas extranjeras debería estar al tanto del lugar que los estudios culturales ocupan en el aula de clase e intentar desarrollar la conciencia intercultural con el fin de mejorar la competencia comunicativa intercultural en sus estudiantes. En este escenario, Shomoosi y Ketabi (2008) recuerdan que la globalización y el rápido esparcimiento del inglés han desafiado las nociones básicas de este idioma y por ende sus prácticas educativas. El objetivo primordial que persigue el aprendizaje del inglés como lenguaje internacional es comunicarse eficientemente con personas de otros contextos; por consiguiente, este debería ser enseñado como una forma de comunicación intercultural (Jenkins, 2006).

En la actualidad, la competencia intercultural se ha convertido en la preocupación principal de docentes que enseñan inglés como lengua extranjera (Liaw, 2006). Al respecto, Stire (2006) considera que la comunicación intercultural no debería ser vista solamente como un encuentro entre culturas; por el contrario, ésta debería ser considerada y analizada como un proceso complejo dentro de la enseñanza del inglés como lengua extranjera.

Por su parte, Chen y Yang (2014) sostienen que el objetivo principal de aprender inglés como lengua extranjera consiste en comunicarse eficientemente con miembros de otras culturas. Para estos autores, anteriormente lo único que muchos docentes de inglés hacían era enseñar el idioma para preparar a los estudiantes a que obtengan buenas calificaciones en los exámenes, mientras para otros el foco era dar a conocer cómo este idioma era utilizado en contextos de habla inglesa. Esto daba lugar a que esta educación no cumpliera con las expectativas reales, las que 
están ligadas a la competencia comunicativa del inglés y que son necesarias en este mundo globalizado. Para Chen y Yang (2014), uno de los modelos más importantes de la competencia comunicativa intercultural es el que propone Byram (1997), quien considera que los objetivos de enseñar una lengua extranjera deben incorporar la competencia intercultural a más de las competencias ya establecidas, como se indicó anteriormente. Es decir, es necesario preparar a los estudiantes para que estén en capacidad de interactuar con personas de otras culturas, comprender $\mathrm{y}$ aceptar individuos de otros contextos culturales por sus valores, puntos de vista $\mathrm{y}$ comportamientos y convertir ésta en una experiencia altamente enriquecedora. Adicionalmente, Byram y Fleming (1998) sostienen que se debería enseñar la cultura el momento que se enseña una lengua extranjera, ya que esto daría lugar a que los estudiantes conozcan sobre la cultura del idioma meta, perspectiva que también es apoyada por McKay (2003).

A lo contrario de lo expresado anteriormente, existe otra perspectiva que cuestiona si es necesaria la enseñanza de la cultura junto con el idioma. Debido a su esparcimiento y empezando con el período de colonización y continuando luego con el poder político de los Estados Unidos, Choudhury indica que el inglés "ha sido utilizado para diferentes propósitos en todas partes del mundo; por ejemplo, en educación, comercio, turismo y en ciencia” (2014, p. 1). Por lo tanto, este autor cuestiona si realmente es necesario enseñar la cultura al momento de enseñar una lengua extranjera, señalando que este tema ha venido debatiéndose por décadas entre académicos, lingüistas y sociolingüistas. En esta misma línea, Kachru (1997), al igual que Seidlhofer (2001), sostiene que debido a que el inglés se ha convertido en lingua franca, no es necesario que la cultura se enseñe cuando se imparte este idioma.

Sin embargo, Tomalin (2008) considera que el rol internacional del inglés y la globalización están íntimamente ligados y se constituyen en dos razones poderosas para incluir la cultura como el quinto elemento del idioma, a más de las destrezas de escuchar, hablar, leer y escribir. Tomalin (2008) también recuerda que:

La quinta destreza del idioma enseña a los estudiantes la actitud y técnica para adaptar su conocimiento del inglés al aprendizaje, comprensión y apreciación de valores, formas diferentes de hacer las cosas y cualidades únicas de otras culturas. También involucra la comprensión de cómo utilizar el idioma para aceptar las diferencias, ser flexible y tolerante con las formas de realizar cosas que pueden ser diferentes a las nuestras. Es un cambio de actitud que se expresa a través del idioma (p. 4).

Por su parte, Dai (2011) sostiene que el mundo hoy en día se está abriendo, dando lugar a que varias culturas se relacionen entre sí, ya que existe mayor intercambio y cooperación mutua. En este escenario, es importante que en la enseñanza de inglés como lengua extranjera los docentes tengan una comprensión clara de la relación entre lengua y cultura con el propósito de cultivar la competencia comunicativa intercultural en sus estudiantes, a la vez que desarrollen nuevas técnicas de enseñanza. Dai (2011) también manifiesta que los docentes de idiomas extranjeros deberían utilizar diferentes métodos en sus clases con el objetivo de ayudar a superar las deficiencias que existen en el aprendizaje del inglés como segunda lengua al igual que en la cultura meta. 


\section{El aprendizaje del inglés en el contexto ecuatoriano}

Con los antecedentes arriba mencionados y sin duda alguna, el inglés es el idioma más utilizado en el mundo; por tanto, su aprendizaje se ha convertido en una necesidad para que las personas conciban el mundo de una forma diferente y establezcan una identidad global nueva (Luo, 2007). Adicionalmente, Cadena Murga et al. (2018) comentan que el uso del inglés es un elemento importante en el proceso de formación de los estudiantes y que "la enseñanza de este idioma ha experimentado cambios significativos debido a que su objetivo ha cambiado mucho, pues se espera que los estudiantes hoy en día se comuniquen eficaz y eficientemente en inglés" (p. 127).

Por su parte, Calle et al. (2012) recuerdan que el Ministerio de Educación del Ecuador, con el propósito de mejorar la enseñanza del idioma inglés en los colegios públicos, inició una reforma curricular en el año 1993, cuyo nombre fue Proyecto Cradle. No obstante, este proyecto no logró su objetivo, pues el rendimiento de los estudiantes no mejoró debido a que los docentes utilizaban métodos tradicionales que no favorecían el desarrollo de la competencia comunicativa. Adicionalmente, cuando los estudiantes llegaban a la universidad, sus conocimientos del idioma inglés no les permitían llegar a niveles intermedios o avanzados. En este escenario, Cadena Murga et al. (2018) mencionan que, luego de la culminación del Proyecto Cradle en el año 2012, el Ministerio de Educación, en la presidencia del Econ. Rafael Correa, inició otra reforma curricular llamada AVANZA, basada en el Marco Común Europeo de Referencia para las Lenguas (MCER). El Ministerio de Educación (2019) ecuatoriano, entre los objetivos establecidos en el Proyecto AVANZA, planteaba que los estudiantes debían alcanzar un nivel B1/2, de acuerdo al MCER, al culminar la secundaria.

Matute (2021) reporta que según el Índice de Dominio del inglés de EF (Education First) (2019), el Ecuador se encuentra entre los países con muy bajo dominio de este idioma, ocupando el puesto 81 en una lista de 100 países. Cuando se analizan los resultados dentro de América Latina, el puntaje de Ecuador es 46,57, lo que lo convierte en el país con el nivel más bajo de inglés de la región. Calle et al. (2012) mencionan que esto se debe a varios factores que son parte de la enseñanza del idioma inglés en el contexto ecuatoriano.

Los profesores fiscales de inglés no emplean estrategias que favorezcan la competencia comunicativa, pues continúan utilizando estrategias tradicionales que obstaculizan el desarrollo de dicha competencia, así como también de las destrezas lingüísticas (leer, escribir, escuchar, hablar) lo que repercute en el bajo rendimiento estudiantil. (p. 2)

Con los antecedentes arriba mencionados, Matute (2021) propone el enfoque de Aprendizaje Integrado de Contenidos y Lenguas (AICLE), Content and Language Integrated Learning (CLIL) en inglés, "un método aplicado en varios países alrededor del mundo que consiste en integrar cualquier asignatura a un lenguaje extranjero con el propósito de adquirir los dos al mismo tiempo" (p. 2). El acrónimo CLIL se acuñó en Europa a principios de la década de los noventa con el propósito de describir cualquier tipo de enfoque dual en el que se utiliza una segunda lengua o lengua extranjera en la enseñanza/aprendizaje de un tema no lingüístico, dando lugar a que el lenguaje y el contenido tengan un papel conjunto y mutuamente beneficioso (Coyle, 2008). Este enfoque goza en estos momentos de enorme popularidad a nivel internacional. Mientras que sus dificultades logísticas y estructurales se reconocen ampliamente, Harrop indica 
que sus limitaciones intrínsecas son objeto de escaso debate (2012, p. 57). Esta autora también sostiene que la flexibilidad del Aprendizaje Integrado de Contenidos y Lenguas está respaldada por un marco teórico comúnmente conocido como el Modelo 4C, que es un enfoque holístico, donde se integran el contenido, la comunicación, la cognición y la cultura. El AICLE efectivo se lleva a cabo a través de cinco dimensiones: progresión en el conocimiento, habilidades y comprensión del contenido, participación en el procesamiento cognitivo de orden superior, interacción en el contexto comunicativo, desarrollo de habilidades de comunicación apropiadas y adquisición de una conciencia intercultural cada vez más profunda (Coyle, 2008).

En este escenario, el enfoque CLIL, implementado en Ecuador, se basa en las ideas de un entorno centrado en el estudiante y mucha interacción entre estudiantes y profesores (Ministerio de Educación, 2016). Finalmente, Matute (2021) menciona que debido a que el AICLE ya es parte del currículo ecuatoriano, las autoridades y los docentes necesitan información más profunda sobre este enfoque para poder utilizarlo, mejorarlo o combinarlo con otras metodologías para potenciar la enseñanza del idioma inglés en los estudiantes.

\section{Conclusiones}

Este trabajo ha tenido el objetivo de presentar una revisión bibliográfica descriptiva de elementos y conceptos relacionados al lenguaje, el inglés como lengua extranjera y su rol como lingua franca dentro de la globalización actual con la intención de complementar y reforzar el conocimiento sobre este tema. Así también, se ha presentado la situación de la enseñanza del inglés como lengua extranjera dentro del contexto educativo ecuatoriano. Como un proceso complejo, no cabe duda que es necesario considerar estos diferentes aspectos para mejorar la práctica docente que permita a los individuos enmarcarse en una sociedad globalizada con las herramientas necesarias para llevar a cabo una comunicación efectiva en variados ámbitos personales y profesionales.

Los individuos hoy en día aprenden y utilizan el idioma inglés no solamente para comunicarse con hablantes de la lengua meta, sino también para comunicarse con personas que pertenecen a otras culturas y que poseen diferentes lenguajes. Por otro lado, se ha vuelto muy común la enseñanza del inglés para la comunicación intercultural, en cuyo caso, el idioma meta es utilizado como lingua franca, convirtiéndose en un término muy de moda en la metodología de la enseñanza de inglés como lengua extranjera. Por lo tanto, como un fenómeno social, la enseñanza y aprendizaje de un idioma extranjero tiene que estar enmarcado dentro de una comprensión intercultural que permita a los interlocutores entablar relaciones más equitativas y de respeto mutuo (Byram, 1977). De esta manera, se estaría dando valor a las cosmovisiones que se reflejan en las interacciones como producto del contacto entre culturas.

Consideramos que este estudio resalta la importancia de los temas tratados. Sin embargo, es necesario que se continúe con debates al respecto y se propicien investigaciones más a fondo que contribuyan con el campo de la enseñanza del inglés como lengua extranjera. Adicionalmente, se podría también hablar de que, en el proceso de comunicación, los hablantes no nativos del inglés son representantes de una cultura particular ya sea local o nacional. En este contexto, al mismo tiempo, valdría la pena preguntarse si los miembros de diferentes culturas pueden comunicarse 
eficientemente a través de una lingua franca sin la necesidad de una apertura intercultural previa que ayude a mantener intercambios más efectivos.

\section{Referencias bibliográficas}

Ballestero, C. y Batista, J. (2007). Evaluación de la enseñanza del inglés con fines específicos en educación superior. Omnia, 13(1),

105-129. https://www.redalyc.org/pdf/737/Resumenes/Resumen_73713106_1.pdf

Beneke, J. (1991). Englisch als lingua franca oder als a medium of intercultural communication. En R. Grebing (Ed.), Grenzenloses Sprachenlernen. 54-66. Cornelsen.

Brown, H. D. (2007). Principles of Language Learning and Teaching (5th ed.). Pearson.

Brumfit, C. J. (2002). Global English and language teaching in the twenty-first century. Centre for Language in Education Occasional Papers No.59, University of Southampton.

Byram, M. (1997). Teaching and Assessing Intercultural Communicative Competence. Multilingual Matters.

Byram, M., \& Flemming, M. (Eds.) (1998). Language Learning from an Intercultural Perspective. Cambridge University Press.

Cadena Murga, B. C., Castillo Niama, M. P., Célleri Quinde, S. P., y Damián Tixi, D. L. (2018). Desarrollo del currículo de inglés como lengua extranjera en el Ecuador. Revista Boletín Redipe, 7(9), 125-139. https://revista.redipe.org/index.php/1/article/view/568

Calle, A., Calle, S., Argudo, J., Moscoso, E., Smith, A., y Cabrera, P. (2012). Los profesores de inglés y su práctica docente: Un estudio de caso de los colegios fiscales de la ciudad de Cuenca, Ecuador. Maskana, 3(2), 1-17. https://doi.org/10.18537/mskn.03.02.01

Chahak, S. M., \& Basirizadeh, F. S. (2012). The study of culture on foreign language teaching. International Journal of Social Science and Humanity, 2(6), 522-524. https://doi.org/10.7763/IJSSH.2012.V2.161

Chen, J. J., \& Yang, S. C. (2014). Fostering foreign language learning through technologyenhanced intercultural projects. Language Learning \& Technology, 18(1), 57-75. https://scholarspace.manoa.hawaii.edu/bitstream/10125/44354/1/18_01_chenyang.pdf

Choudhury, R. U. (2014). The role of culture in teaching and learning of English as a foreign language. Express, an International Journal of Multi-Disciplinary Research, 1(4), 1-20.

Coyle, D. (2008). Content and Language Integrated Learning: Towards a connected research agenda for CLIL pedagogies. International Journal of Bilingual Education and Bilingualism, 10(5), 543-562. https://doi.org/10.2167/beb459.0

Dai, L. (2011). Practical Techniques for Cultural-based Language Teaching in the EFL Classroom. Journal of Language Teaching \& Research,2(5), 1031-1036. https://doi.org/10.4304/JLTR.2.5.1031-1036

Day, R. A. (2005). Cómo escribir y publicar trabajos científicos (M. Sáenz, Trans; 3era. ed.). (Publicación Científica y Técnica No. 598). Organización Panamericana de la Salud. https://bit.ly/3BhbSUq

Dearden, J. (2014). English as a medium of instruction - a growing global phenomenon. British Council [Archivo PDF]. https://www.britishcouncil.org/sites/default/files/e484_emi_cover_option_3_final_web.pdf

Díaz-Aguado, M. J. (2003). Educación Intercultural y aprendizaje cooperativo. Pirámide. 
Dimas, H. M. S. (2016). Integrating the Intercultural Communicative Competence (ICC) in a Foreign Language Program: Faculty Considerations upon Leaving the Haven of Native Speakership. English Language Teaching, 9(4), 1-10. https://doi.org/10.5539/elt.v9n4p1

Douglas, D. (2014). Nobody seems to speak English here today: Enhancing assessment and training in aviation English. Iranian Journal of Language Teaching Research, 2(2), 1-12. https://files.eric.ed.gov/fulltext/EJ1127407.pdf

Duranti, A. (1989). Intentions, Language and Social Action in Samoan Context. Journal of Pragmatics, 12, 13-33.

EF Education First (2019). English Proficiency Index: A Ranking of 100 Countries and Regions by English Skills (9 ${ }^{\text {th }}$ ed.). https://www.ef.com/wwen/epi/

Firth, A. (1996). The discursive accomplishment of normality: On 'lingua franca' English and conversation analysis. Journal of Pragmatics, 26(2), 237-259. https://doi.org/10.1016/0378-2166(96)00014-8

Gao, F. (2006). Language is Culture. On Intercultural Communication. Journal of Language and Linguistics, 5(1), 58-67.

Graddol, D. (1997). The future of English? A guide to forecasting the popularity of the English language in the 21st century. British Council.

Harrop, E. (2012). Content and language integrated learning (CLIL): Limitations and possibilities. Encuentro, 21, 57-70. https://files.eric.ed.gov/fulltext/ED539731.pdf

House, J. (1999). Misunderstanding in intercultural communication. Interactions in English as a lingua franca and the myth of mutual intelligibility. In C. Gnutzmann (Ed.), Teaching and learning English as a global language. 37-89. Stauffenburg.

Hymes, D. (1972). The Uses of Anthropology: Critical, Political, Personal. En D. Hymes (Ed.), Reinventing Anthropology. Random House.

Jenkins, J. (2006). Current Perspectives on Teaching World Englishes and English as a Lingua Franca. TESOL Quarterly, 40(1), 157-181. https://doi.org/10.2307/40264515

Kachru, B. (1985). Standards, codification, and sociolinguistic realism: The English Language in the outer circle. En R. Quirk y H. Widdowson (Eds.), English in the world: Teaching and learning the language and literatures. 11-30. Cambridge University Press.

Kachru, B. (1992). The Other Tongue. English Across Cultures (2nd ed.). University of Illinois Press.

Kumaravadivelu, B. (2008). Cultural Globalization and Language Education. Yale University Press.

Larsen-Freeman, D., \& Freeman, D. (2008). Language moves: The place of "foreign" languages in classroom teaching and learning. Review of Research in Education, 32(1), 147-186. https://doi.org/10.3102/0091732X07309426

Liaw, M. (2006). E-learning and the development of intercultural competence. Language Learning \& Technology, 10(3), 49-64. https://scholarspace.manoa.hawaii.edu/bitstream/10125/44074/1/10_03_liaw.pdf

López, M. (2006). Cultura de la diversidad, cultura de la inclusión: educar para construir una escuela sin exclusiones. En Actas de las XVI Jornadas Municipales de Psicopedagogía “L'Ecola que inclou”, España, 18, 11- 52.

Luo, W. (2007). English language teaching in Chinese Universities in the era of the World Trade Organization: A learner perspective. [Tesis doctoral no publicada]. RMIT University. https://core.ac.uk/download/pdf/15618217.pdf 
Matute, M. (2021). Effects of Using Content and Language Integrated Learning Instruction in English as a Foreign Language Classes. [Tesis de licenciatura no publicada]. Universidad de Cuenca.

McKay, S. (2002). Teaching English as an international language: Rethinking goals and approaches. Oxford University Press.

McKay, S. (2003). Teaching English as an international language: The Chilean context. ELT Journal, 57(2), 139-48.

Méndez García, M.C. (2013). The intercultural turn brought about by the implementation of CLIL programmes in Spanish monolingual areas: a case study of Andalusian primary and secondary schools. The Language Learning Journal, 41(3), 268-283. https://doi.org/10.1080/09571736.2013.836345

Menéndez, J. D. (2003). La gestión de la cooperación internacional en las universidades. Revista Cubana de Educación Superior, 23(1), 47-68.

Merriam-Webster's Collegiate Dictionary (11th ed.). (2003). Merrian-Webster.

Ministerio de Educación. (2019). Currículo de los Niveles de Educación Obligatoria: Nivel Bachillerato (2da. ed., vol. 1). Ministerio de Educación del Ecuador.

Pinker, S. (1994). The language instinct: How the mind creates language. William Morrow.

Samarin, W. (1987). Lingua franca. En U. Ammon, N. Dittman, y K. Mattheier (Eds.), Sociolinguistics: An international handbook of the science of language and society (pp. 371-374). Walter de Gruyter.

Scollon, R. (2004). Teaching language and culture as hegemonic practice. The Modern Language Journal, 88(2), 271-274. https://www.jstor.org/stable/3588757

Seidlhofer, B. (2004). 10. Research perspectives on teaching English as a lingua franca. Annual Review of Applied Linguistics, 24, 209-239. https://doi.org/10.1017/S0267190504000145

Seidlhofer, B. (2001). Closing a conceptual gap: the case for a description of English as a lingua franca. International Journal of Applied Linguistics, 11(2), 133-158. https://doi.org/10.1111/1473-4192.00011

Shomoossi, N., \& Ketabi, S. (2008). Authenticity within the EIL Paradigm. Iranian Journal of Language Studies, 2(2), 173-185.

Stire, J. (2006). Internationalisation, intercultural communication and intercultural competence. Journal of Intercultural Communication, $11(1), \quad 1-12$. https://www.immi.se/intercultural/nr11/stier.pdf

Tomalin, B. (2008, September 29). Culture - the fifth language skill. British Council http://www.teachingenglish.org.uk/articles/culture-fifth-language-skill

Van Weijen, D. (2012). The Language of (Future) Scientific Communication. Research Trends, 31. https://bit.ly/3zwDHYr

Wei, Y. (2005). Integrating Chinese Culture with TEFL in Chinese Classroom. Sino-US English Teaching, 2(7), 55-58. 